\title{
STEINER'S FORMULA IN THE HEISENBERG GROUP
}

\author{
ZOLTÁN M. BALOGH, FAUSTO FERRARI, BRUNO FRANCHI, \\ EUGENIO VECCHI AND KEVIN WILDRICK
}

\begin{abstract}
Steiner's tube formula states that the volume of an $\epsilon$-neighborhood of a smooth regular domain in $\mathbb{R}^{n}$ is a polynomial of degree $n$ in the variable $\epsilon$ whose coefficients are curvature integrals (called also as quermassintegrals). We prove a similar result in the sub-Riemannian setting of the first Heisenberg group. In contrast to the Euclidean setting, we find that the volume of an $\epsilon$-neighborhood with respect to the Heisenberg metric is an analytic function of $\epsilon$ that is generally not a polynomial. The coefficients of the series expansion can be explicitly written in terms of integrals of iteratively defined canonical polynomials of just five curvature terms.
\end{abstract}

\section{CONTEnTs}

1. Introduction 1

2. Notation and basic results 4

3. The derivatives of the volume function 5

3.1. The construction and properties of the localizing set Q 5

3.2. Derivatives and iterated divergences $\quad 8$

4. Calculating the iterated divergences 10

4.1. A recursive formula $\quad 10$

4.2. Analyticity of the volume function $\quad 14$

5. An example and final remarks $\quad 15$

\section{INTRODUCTION}

Let us denote by $\Omega \subseteq \mathbb{R}^{n}$ a bounded regular domain in Euclidean space, and by $\Omega_{\epsilon}$ its $\epsilon$ neighborhood with respect to the usual Euclidean metric. The celebrated Steiner's formula expresses the volume $\operatorname{vol}\left(\Omega_{\epsilon}\right)$ as a polynomial in $\epsilon$

$$
\operatorname{vol}\left(\Omega_{\epsilon}\right)=\sum_{k=0}^{n} a_{k} \epsilon^{k},
$$

where the coefficients $a_{k}$ are the so called quermassintegrals of $\Omega$.

This formula goes back to J. Steiner who proved it in two and three dimensional Euclidean spaces for convex polytopes. It has been generalized later by H. Weyl to the setting of arbitrary smooth submanifolds of $\mathbb{R}^{n}$. We refer the interested reader to the monograph of A. Gray [?] for an exhaustive overview of this subject, as well as [?]. A localized version of the above formula still holds even for non-smooth submanifolds as shown by H. Federer [?]. Recently these notions have

Key words and phrases. Heisenberg group, Steiner's formula 2010 Mathematics Subject Classification: $43 A 80$.

F.F. is supported by the ERC starting grant project 2011 EPSILON (Elliptic PDEs and Symmetry of Interfaces and Layers for Odd Nonlinearities) 277749 and by RFO grant, Università di Bologna, Italy. B.F. is supported by GNAMPA of INdAM, Italy, and by University of Bologna, funds for selected research topics, Z.M.B., E.V. and K.W. supported by the Swiss National Science Foundation. 
been widely used to obtain new results concerning nonlinear PDEs and Sobolev inequalities, see e.g. [?], [?] and [?], and relative isoperimetric inequalities, see [?].

The purpose of this paper is to prove a similar result also in the sub-Riemannian setting of the first Heisenberg group $\mathbb{H}$. Indeed, it is well known that $\mathbb{H}$ can be endowed with its canonical left-invariant Carnot-Carathéodory metric, and therefore it is natural to search for a formula akin to (1.1), where the $\epsilon$-neighborhood $\Omega_{\epsilon}$ should be replaced by an $\epsilon$-neighborhood with respect to the Carnot-Carathéodory metric (basic notation and results about the metric structure of the Heisenberg group can be found in Section 2). This interest is motivated by the recent progress in the geometric measure theory of Lie groups (e.g.[?, ?, ?, ?, ?, ?]). In the aforementioned papers, many tools of the Euclidean theory related to rectifiability and perimeter, such as co-area and divergence formulae, have been developed in the sub-Riemannian setting of non-commutative Lie groups. Nevertheless, the notions of higher order curvatures even in the simplest instance of $\mathbb{H}$ are still far from being fully understood. We hope this paper can provide some hints in this direction. For a general overview of these results we refer to the monograph [?].

Our approach is inspired by the work of R.C. Reilly [?], [?] which is based on expressing the coefficients $a_{k}$ in Steiner's formula (1.1) in terms of integrals of iterated divergences of the signed Carnot-Carathéodory distance function $\delta$ associated to $\Omega$.

In our setting of the first Heisenberg group, instead of the full divergence, we consider the so called horizontal divergence of a horizontal vector field $X=u_{1} X_{1}+u_{2} X_{2}$ where $X_{1}$ and $X_{2}$ are the canonical left-invariant horizontal vector fields in $\mathbb{H}$ and $u_{1}$ and $u_{2}$ are arbitrary smooth functions.

In this situation, the horizontal divergence of $X$ is given by $\operatorname{div}_{\mathrm{H}} X:=X_{1} u_{1}+X_{2} u_{2}$. If $u$ is a smooth function in an open set of $\mathbb{H}$ we shall consider the iterated horizontal divergences of $u$ according to the relations:

$$
\operatorname{div}_{\mathrm{H}}{ }^{(0)} \nabla_{H} u=1, \operatorname{div}_{\mathrm{H}}{ }^{(i)} \nabla_{\mathrm{H}} u=\operatorname{div}_{\mathrm{H}}\left(\left(\operatorname{div}_{\mathrm{H}}{ }^{(i-1)} \nabla_{H} u\right) \cdot \nabla_{H} u\right), \quad i \geq 1,
$$

where $\nabla_{H} u:=\left(X_{1} u\right) X_{1}+\left(X_{2} u\right) X_{2}$ is the horizontal gradient of $u$. With this notation our first statement reads as follows:

Theorem 1.1. Let $\Omega \subseteq \mathbb{H}$ be a bounded smooth domain with $\mathcal{C}^{\infty}$-regular boundary and $Q \subseteq \mathbb{H}$ be a localizing set with the property that $\partial \Omega \cap Q$ is free from characteristic points. We denote by $\delta$ the signed Carnot-Carathédory distance function defined in a neighborhood of $\partial \Omega \cap Q$.

For $\epsilon \geq 0$, let $\Omega_{\epsilon} \cap Q$ be a localized Heisenberg $\epsilon$-neighborhood of $\Omega$. Then the function $\epsilon \mapsto \operatorname{vol}\left(\Omega_{\epsilon} \cap Q\right)$ is real-analytic, and has a power series expansion given by

$$
\operatorname{vol}\left(\Omega_{\epsilon} \cap Q\right)=\operatorname{vol}(\Omega \cap Q)+\sum_{i=1}^{\infty} a_{i} \frac{\epsilon^{i}}{i !},
$$

where

$$
a_{i}=\int_{\partial \Omega \cap Q}\left(\operatorname{div}_{\mathrm{H}}{ }^{(i-1)} \nabla_{H} \delta\right) d \mathcal{H}_{d_{c c}}^{3} .
$$

The remarkable fact is that, although the $(i-1)^{\text {st }}$ iterated divergence, $i>1$, of a smooth function $u$ contains, a priori, derivatives of order $i$, for the signed distance function $\delta$ this is not the case. It turns out that all coefficients $a_{i}$ appearing in Theorem 1.1 are integrals of polynomials of certain second order derivatives of the function $\delta$.

To simplify the notation for iterated applications of the vector fields $X_{i}, i=1,2$ we will use for $X_{i}\left(X_{j}\right)$ the notation $X_{i j}$. 
The main result of our paper gives a precise recursive formula for the iterated divergences in terms of the following quantities:

$$
\begin{gathered}
A:=\Delta_{\mathrm{H}} \delta:=X_{11} \delta+X_{22} \delta, \quad B:=-\left(4 X_{3} \delta\right)^{2}, \quad C:=-4\left(\left(X_{1} \delta\right)\left(X_{32} \delta\right)-\left(X_{2} \delta\right)\left(X_{31} \delta\right)\right), \\
D:=16 X_{33} \delta, \quad E:=16\left(\left(X_{31} \delta\right)^{2}+\left(X_{32} \delta\right)^{2}\right) .
\end{gathered}
$$

Theorem 1.2. Under the conditions of Theorem 1.1, the following relations hold:

$$
\begin{gathered}
\operatorname{div}_{\mathrm{H}}^{(1)} \nabla_{H} \delta=A, \quad \operatorname{div}_{\mathrm{H}}{ }^{(2)} \nabla_{H} \delta=B+2 C, \\
\operatorname{div}_{\mathrm{H}}^{(3)} \nabla_{H} \delta=A B+2 D, \quad \operatorname{div}_{\mathrm{H}}{ }^{(4)} \nabla_{H} \delta=B^{2}+2 B C+2 A D-2 E,
\end{gathered}
$$

and for all $j \geq 2$,

$$
\begin{aligned}
\operatorname{div}_{\mathrm{H}}{ }^{(2 j-1)} \nabla_{H} \delta & =B^{j-2}(A B+2(j-1) D), \\
\operatorname{div}_{\mathrm{H}}{ }^{(2 j)} \nabla_{H} \delta & =B^{j-2}\left(B^{2}+2 B C+2(j-1)(A D-E)\right) .
\end{aligned}
$$

Generally speaking, it is feasible to think that the integrals of iterated horizontal divergences appearing in the above expressions should carry important geometric information about the Heisenberg geometry of the domain $\Omega$. In particular the expression

$$
\operatorname{div}_{\mathrm{H}}^{(1)} \nabla_{H} \delta=\Delta_{\mathrm{H}} \delta:=X_{11} \delta+X_{22} \delta
$$

is currently the accepted notion of the horizontal mean curvature of $\partial \Omega$, and indeed of the level sets $\{\delta=\epsilon\}$ for sufficiently small values of $\epsilon$ [?]. This notion of mean curvature plays a crucial role in the study of minimal surfaces in the Heisenberg group.

Analogously, the expression

$$
\operatorname{div}_{\mathrm{H}}^{(2)} \nabla_{H} \delta=-\left(4 X_{3} \delta\right)^{2}-8\left(\left(X_{1} \delta\right)\left(X_{32} \delta\right)-\left(X_{2} \delta\right)\left(X_{31} \delta\right)\right)
$$

may provide a useful notion of theof a surface in the Heisenberg group. While the above expression has not yet been investigated in depth, recent results [?], indicate however that this formula comes out as the limit of the sectional curvature of a surface in the Riemannian approximation of the Heisenberg group and gives an appropriate version of the Gauss-Bonnet theorem in the Heisenberg setting.

Some further information about the existing literature is now in order. By homogeneity, it is easy to prove that Steiner's formula for a Carnot-Carathéodory ball in the Heisenberg group is a polynomial of degree 4; in [?], the coefficients of this polynomial have been explicitly found, computing the flow of the horizontal gradient of the signed distance function; a track of this flow is called a metric normal, the theory of which has been developed in [?], [?]. While this approach does not relate the iterated divergences to volume nor allow for localization, it is very effective in computing the volume function for explicit sets.

The paper is structured as follows: in Section 2 notations are fixed and background results of the Heisenberg calculus are recalled. Section 3 is devoted to a careful analysis of localizing sets and the link between the derivatives of the volume function and the integrals of the iterated divergences is established. In Section 4 we prove the recursive formulae stated in Theorem 1.2 and Theorem 1.1. In Section 5 we present an example in which Steiner's formula is not a polynomial and where the coefficients in Theorem 1.1 can be computed explicitly.

Acknowledgements. We thank the referee for carefully reading the paper and for all the useful comments and suggestions to improve the style and the content of the paper. 


\section{Notation AND BASiC RESUlts}

Given points $x=\left(x_{1}, x_{2}, x_{3}\right)$ and $x^{\prime}=\left(x_{1}^{\prime}, x_{2}^{\prime}, x_{3}^{\prime}\right)$ in $\mathbb{R}^{3}$, the Heisenberg product is given by

$$
x * x^{\prime}=\left(x_{1}+x_{1}^{\prime}, x_{2}+x_{2}^{\prime}, x_{3}+x_{3}^{\prime}+2\left(x_{2} x_{1}^{\prime}-x_{1} x_{2}^{\prime}\right),\right.
$$

defining the Heisenberg group $\mathbb{H}$. The corresponding Lie algebra is generated by the left-invariant vector fields

$$
X_{1}=\frac{\partial}{\partial x_{1}}+2 x_{2} \frac{\partial}{\partial x_{3}}, X_{2}=\frac{\partial}{\partial x_{2}}-2 x_{1} \frac{\partial}{\partial x_{3}}, X_{3}=\frac{\partial}{\partial x_{3}} .
$$

We employ this somewhat unusual notation for the readability of the computations to be made in Section 4.

The horizontal distribution

$$
H \mathbb{H}:=\operatorname{span}\left\{X_{1}, X_{2}\right\} \subseteq T \mathbb{R}^{3}
$$

is equipped with the inner product $\langle\cdot, \cdot\rangle_{\mathrm{H}}$ in which $X_{1}$ and $X_{2}$ form an orthonormal basis. This induces the horizontal norm $\|\cdot\|_{\mathrm{H}}$.

Since $\left[X_{1}, X_{2}\right]=-4 X_{3}$, the horizontal distribution $H \mathbb{H}$ is non-integrable. It follows that any pair of points $x, x^{\prime} \in \mathbb{H}$ can be connected by an absolutely continuous curve $\gamma:[0,1] \rightarrow \mathbb{R}^{3}$ with the property that $\gamma^{\prime}(s) \in H_{\gamma(s)} \mathbb{H}$ for almost every $s \in[0,1]$; such a curve is called horizontal. Measuring the length of horizontal curves by using $\|\cdot\|_{\mathrm{H}}$ results in the Carnot-Carathéodory metric on $\mathbb{H}$, which is denoted

$$
d_{c c}\left(x, x^{\prime}\right):=\inf \left\{\int_{\gamma}\left\|\gamma^{\prime}(s)\right\|_{\mathrm{H}} d s: \gamma \text { is a horizontal curve connecting } x \text { to } x^{\prime}\right\} .
$$

The Haar measure on $\mathbb{H}$, the 3 -dimensional Lebesgue measure $\mathcal{L}^{3}$, and the 4-dimensional Hausdorff

measure $\mathcal{H}_{d_{c c}}^{4}$ of the metric $d_{c c}$ all coincide up to a scaling. We will most often employ the Lebesgue measure.

Throughout this paper, we will work with the following standing assumptions and notations:

(1) We consider a fixed but arbitrary open set $\Omega \subseteq \mathbb{R}^{3}$ whose boundary $\partial \Omega$ is a $\mathcal{C}^{\infty}$-smooth surface.

(2) The signed distance of a point $g \in \mathbb{H}$ from $\partial \Omega$ is denoted by $\delta: \mathbb{H} \rightarrow[0, \infty)$, where

$$
\delta(g)= \begin{cases}\operatorname{dist}_{c c}(g, \partial \Omega) & g \in \mathbb{H} \backslash \Omega, \\ -\operatorname{dist}_{c c}(g, \partial \Omega) & g \in \bar{\Omega} .\end{cases}
$$

(3) The characteristic set of $\partial \Omega$ is defined by

$$
\operatorname{char}(\partial \Omega)=\left\{g \in \partial \Omega: T_{g} \partial \Omega=H_{g} \mathbb{H}\right\} .
$$

This set is pathological from the perspective of the regularity of the distance function $\delta$. We consider an arbitrary bounded, connected, and relatively open set $U_{0} \subseteq \partial \Omega$ with the property that

$$
\operatorname{dist}_{c c}\left(U_{0}, \operatorname{char}(\partial \Omega)\right)>0 .
$$

A basic result of [?] implies that there is a connected, bounded, and open set $U \subseteq \mathbb{R}^{3}$ that contains $\bar{U}_{0}$ and on which $\delta$ has one degree of regularity less than $\partial \Omega$. Hence, we will have that $\delta$ is $\mathcal{C}^{\infty}$-smooth on $U_{0}$.

(4) The Euclidean gradient field $\nabla \delta: U \rightarrow \mathbb{R}^{3}$ is non-vanishing and normal (in the Euclidean sense) to the level set $\delta^{-1}(\epsilon)$ near any point of $U$. The projection of this vector field onto the horizontal distribution $H \mathbb{H}$ yields the embedded horizontal normal $N: U \rightarrow \mathbb{R}^{3}$ defined by

$$
N=\left(X_{1} \delta\right) X_{1}+\left(X_{2} \delta\right) X_{2}
$$


The basis of this work is the fact that the signed distance function satisfies the eikonal equation in the following sense (see [?]):

$$
\|N(g)\|_{\mathrm{H}}=1, \quad \text { for } \mathcal{L}^{3} \text {-almost every } g \in U .
$$

In fact, the smoothness of $\delta$ implies that $N$ is also $\mathcal{C}^{\infty}$-smooth, and so (2.2) holds everywhere on $U$. It follows that the Euclidean gradient $\nabla \delta$ does not vanish at any point of $U$. Therefore, $\delta$ can be considered as a defining function for the level set $\delta^{-1}(\epsilon)$ near any point of $U$. This implies that these level sets are $\mathcal{C}^{\infty}$-smooth near any point of $U$.

(5) Given a differentiable function $\alpha: U \rightarrow \mathbb{R}$, we define the horizontal gradient of $\alpha$ to be the projection of the Euclidean gradient of $\alpha$ onto the horizontal distribution, i.e., $\nabla_{\mathrm{H}} \alpha: U \rightarrow \mathbb{R}^{3}$ is given by

$$
\nabla_{\mathrm{H}} \alpha=\left(X_{1} \alpha\right) X_{1}+\left(X_{2} \alpha\right) X_{2} .
$$

Note that we have defined $\nabla_{\mathrm{H}} \alpha$ to be a vector field in $\mathbb{R}^{3}$, and not as the two-dimensional vector field $\left(X_{1} \alpha, X_{2} \alpha\right): U \rightarrow \mathbb{R}^{2}$, as is often the case. In particular, $N=\nabla_{\mathrm{H}} \delta$. We will use both notations to denote this object: $N$ will be employed when its role is geometric in nature, and $\nabla_{\mathrm{H}} \delta$ will be employed when its role is more analytic in nature.

(6) Let $V=a X_{1}+b X_{2}: U \rightarrow \mathbb{R}^{3}$ be a differentiable vector field with values in the horizontal distribution. A key role in this paper is played by the horizontal divergence of $V$, which is defined by

$$
\operatorname{div}_{\mathrm{H}} V=X_{1} a+X_{2} b \text {. }
$$

\section{The DeRIVATIVES OF THE VOLUME FUnCTION}

3.1. The construction and properties of the localizing set $\mathbf{Q}$. If $\Omega$ is unbounded, the volume of its Heisenberg $\epsilon$-neighborhood is infinite. To avoid this, we consider a localized version of the volume function. In the setting of $\mathbb{R}^{3}$, this can be done as follows. One assumes that $\bar{\Omega}$ has positive reach, meaning that there is a number $r>0$ such that if $\operatorname{dist}_{\mathbb{R}^{3}}(x, \bar{\Omega})<r$, then there is a unique point $\pi_{\bar{\Omega}}(x) \in \bar{\Omega}$ of minimal distance to $x$. For each bounded Borel subset $Q \subseteq \mathbb{R}^{3}$ and $\epsilon \in[0, r)$, one considers the set

$$
\mathcal{T}(Q, \bar{\Omega}, \epsilon)=\left\{x \in \mathbb{R}^{3}: \operatorname{dist}_{\mathbb{R}^{3}}(x, \bar{\Omega}) \leq \epsilon \text { and } \pi_{\bar{\Omega}}(x) \in Q\right\}
$$

and seeks a Taylor series expansion of the function

$$
\epsilon \mapsto \mathcal{L}^{3}(\mathcal{T}(Q, \bar{\Omega}, \epsilon))
$$

at $\epsilon=0$.

The requirement that $\bar{\Omega}$ have positive reach is far weaker than our assumptions on $\Omega$. Since we have assumed that $\partial \Omega$ is $\mathcal{C}^{\infty}$-smooth, we may view the set $\mathcal{T}(Q, \bar{\Omega}, \epsilon)$ above as the union of $\bar{\Omega} \cap Q$ and the tracks of $\partial \Omega \cap Q$ under the gradient flow of the Euclidean distance-to- $\partial \Omega$ function $\operatorname{dist}_{\mathbb{R}^{3}}(\cdot, \partial \Omega)$ for time $\epsilon$. This gradient flow can also be viewed as the flow associated to the Euclidean outward-pointing normal to the level sets of $\operatorname{dist}_{\mathbb{R}^{3}}(\cdot, \partial \Omega)$. The volume of $\bar{\Omega} \cap Q$ is the constant term of the desired Taylor series.

It is (roughly) this later approach that we will adapt to the Heisenberg setting. Instead of considering an arbitrary Borel set $Q \subseteq \mathbb{R}^{3}$ for localization, we begin with any sufficiently regular set $B_{0} \subseteq U_{0} \subseteq(\partial \Omega \backslash \operatorname{char}(\partial \Omega))$ and define the localizing set $Q$ to be the image of the flow associated with the embedded horizontal normal $N$. As mentioned in the introduction, this flow has been studied in depth as the metric normal in [?] and [?].

We now implement the above approach. For simplicity, we consider $B_{0} \subseteq U_{0} \subseteq \partial \Omega$ to be of the form

$$
B_{0}:=\bar{B}_{\mathbb{R}^{3}}(p, r) \cap \partial \Omega,
$$


where $p \in \mathbb{H}$ and $r>0$ are chosen so that $B_{0}$ is homeomorphic to a closed disk; this situation generalizes easily to the situation that $B_{0}$ is the closure of any connected open subset of $U_{0}$ with Lipschitz boundary components.

Because of this simplification, we may parametrize the boundary $\partial B_{0}$ of $B_{0}$ with a single smooth function

$$
\beta:[-\tau, \tau] \longrightarrow \partial B_{0}
$$

for some $\tau>0$.

The following proposition states that the flow of the embedded horizontal normal exists on any short time interval containing 0 .

Proposition 3.1. There exists $s_{0}>0$ such that for any $g_{0} \in U_{0} \subseteq \partial \Omega$, the Cauchy problem

$$
\left\{\begin{array}{l}
\dot{\varphi}(s)=N(\varphi(s)), \\
\varphi(0)=g_{0} \in U_{0},
\end{array}\right.
$$

has a local solution $\varphi_{g_{0}}:\left[-s_{0}, s_{0}\right] \rightarrow U$ satisfying

$$
d_{c c}\left(g_{0}, \varphi_{g_{0}}(\sigma)\right) \leq|\sigma| \quad \text { and } \quad \delta\left(\varphi_{g_{0}}(\sigma)\right)=\sigma
$$

for each $\sigma \in\left[-s_{0}, s_{0}\right]$.

Remark 3.2. The inequality in (3.2) becomes an equality if and only if the local solution $\varphi_{g_{0}}$ is a geodesic.

We define the localizing set of depth $s_{0}$ generated by the set $B_{0} \subseteq U_{0} \subseteq \partial \Omega$ by

$$
Q:=\left\{\varphi_{g}(s): g \in B_{0},|s| \leq s_{0}\right\} .
$$

For $0 \leq \epsilon \leq s_{0}$, we consider the localized Heisenberg $\epsilon$-tube

$$
\mathcal{T}_{\mathbb{H}}\left(B_{0}, \bar{\Omega}, \epsilon\right):=\left\{g \in Q:-s_{0} \leq \delta(g) \leq \epsilon\right\}
$$

and we seek to give a Taylor series expansion of the function

$$
\epsilon \mapsto \mathcal{L}^{3}\left(\mathcal{T}_{\mathbb{H}}\left(B_{0}, \bar{\Omega}, \epsilon\right)\right)
$$

near $\epsilon=0$. Note that we may write $\mathcal{T}_{\mathbb{H}}\left(B_{0}, \bar{\Omega}, \epsilon\right)$ as the disjoint union

$$
\mathcal{T}_{\mathbb{H}}\left(B_{0}, \bar{\Omega}, \epsilon\right)=(\bar{\Omega} \cap Q) \cup Q_{\varepsilon},
$$

where

$$
Q_{\epsilon}:=\{g \in Q: 0<\delta(g)<\varepsilon\}
$$

is the track of $B_{0}$ under the flow of the embedded horizontal normal $N$ for positive time $\epsilon$. Hence, our localization procedure is in direct analogy with the Euclidean case. As in the Euclidean case, the volume of $\bar{\Omega} \cap Q$ will be the constant term of the desired Taylor series, and so we will be mostly concerned with estimating the volume of $Q_{\epsilon}$.

The key tool in doing so is a version of the divergence theorem adapted to the structure of the Heisenberg group and of our localizing set $Q$. For this we will need to identify the boundary of certain sets related to $Q_{\epsilon}$. For $-s_{0}<s<t<s_{0}$, denote

$$
Q_{s, t}:=\{g \in Q: s<\delta(g)<t\}=\delta^{-1}((s, t)) \cap Q,
$$


so that $Q_{\epsilon}=Q_{0, \epsilon}$. We define the initial boundary, the lateral boundary, and the final boundary of $Q_{s, t}$ by

$$
\begin{aligned}
\partial_{i} Q_{s, t} & :=\left\{\varphi_{g}(s), g \in B_{0}\right\}=\delta^{-1}(s) \cap Q, \\
\partial_{l} Q_{s, t} & :=\left\{\varphi_{g}(\epsilon): g \in \partial B_{0}, s<\epsilon<t\right\}, \\
\partial_{f} Q_{s, t} & :=\left\{\varphi_{g}(t), g \in B_{0}\right\}=\delta^{-1}(t) \cap Q .
\end{aligned}
$$

respectively. An elementary argument shows that

$$
\partial\left(Q_{s, t}\right)=\partial_{i} Q_{s, t} \cup \partial_{l} Q_{s, t} \cup \partial_{f} Q_{s, t} .
$$

Define a vector field $\mu: \partial\left(Q_{s, t}\right) \rightarrow \mathbb{R}^{3}$ by

$$
\mu(p)= \begin{cases}-\frac{\nabla \delta(p)}{\|\nabla \delta(p)\|_{\mathbb{R}^{3}}} & p \in \partial_{i} Q_{s, t}, \\ w(p) & p \in \partial_{l} Q_{s, t}, \\ \frac{\nabla \delta(p)}{\|\nabla \delta(p)\|_{\mathbb{R}^{3}}} & p \in \partial_{f} Q_{s, t},\end{cases}
$$

where $w: \partial_{l} Q_{s, t} \rightarrow \mathbb{R}^{3}$ is the Euclidean outward unit normal vector to $\partial\left(Q_{s, t}\right)$. Then $\mu$ is the Euclidean unit outward-pointing normal vector field to $\partial\left(Q_{s, t}\right)$. Denote its projection onto the horizontal distribution by $\mu_{\mathrm{H}}$, so that

$$
\mu_{\mathrm{H}}(p)= \begin{cases}-\frac{N(p)}{\|\nabla \delta(p)\|_{\mathbb{R}^{3}}} & p \in \partial_{i} Q_{s, t}, \\ w_{H}(p) & p \in \partial_{l} Q_{s, t}, \\ \frac{N(p)}{\|\nabla \delta(p)\|_{\mathbb{R}^{3}}} & p \in \partial_{f} Q_{s, t},\end{cases}
$$

where $w_{\mathrm{H}}$ is the projection of $w$ onto the horizontal distribution.

The next result shows that on the lateral boundary, the vector $w_{\mathrm{H}}$ is perpendicular to the embedded horizontal normal $N$ with respect to the scalar product $\langle\cdot, \cdot\rangle_{\mathrm{H}}$. The analogous result in the Euclidean case is obvious.

Lemma 3.3. Let $p \in \partial_{l} Q_{s, t}$. Then

$$
\left\langle N(p), w_{\mathrm{H}}(p)\right\rangle_{\mathrm{H}}=0 .
$$

Proof. Recall that we have already parametrized $B_{0}$ by the smooth function $\beta:[-\tau, \tau] \rightarrow \partial B_{0}$. Therefore, a parametrization of the lateral boundary $\partial_{l} Q_{\epsilon}$ is given by $\psi:[-\tau, \tau] \times(0, \epsilon)$, where

$$
\psi(t, s)=\varphi_{\beta(t)}(s) .
$$

The Euclidean tangent space to $\partial_{l} Q_{\epsilon}$ at a point $p=\left(p_{1}, p_{2}, p_{3}\right)=\psi\left(t_{0}, s_{0}\right)$ is thus spanned by

$$
\begin{aligned}
v=\left(v_{1}, v_{2}, v_{3}\right) & :=\frac{\partial \psi}{\partial t}\left(t_{0}, s_{0}\right), \text { and } \\
N(p) & =\frac{\partial \psi}{\partial s}\left(t_{0}, s_{0}\right) .
\end{aligned}
$$

For convenience, denote $N(p)=n_{1} X_{1}(p)+n_{2} X_{2}(p)$. Taking the cross product of $v$ and $N(p)$ now shows that $w(p)$ is a multiple of $\left(w_{1}, w_{2}, w_{3}\right)$, where

$$
\begin{aligned}
& w_{1}=2 v_{2}\left(p_{2} n_{1}-p_{1} n_{2}\right)-v_{3} n_{2} \\
& w_{2}=-2 v_{1}\left(p_{2} n_{1}-p_{1} n_{2}\right)+v_{3} n_{1} \\
& w_{3}=v_{1} n_{2}-v_{2} n_{1} .
\end{aligned}
$$


The projection $w_{\mathrm{H}}(p)$ of $\left(w_{1}, w_{2}, w_{3}\right)$ onto the horizontal tangent space $H_{p} \mathbb{H}$ is given by

$$
\left(w_{1}+2 p_{2} w_{3}\right) X_{1}(p)+\left(w_{2}-2 p_{1} w_{3}\right) X_{2}(p) .
$$

The result now follows from a simple calculation.

Now, we recall that away from the characteristic set, the Heisenberg surface measure is mutually absolutely continuous with respect to the Euclidean 2-dimensional surface measure, and the RadonNikodym derivative is precisely the Euclidean length of the gradient of $\delta$. The relevant result is given in [?], which in our setting translates to the following: for any $s \in\left[-s_{0}, s_{0}\right]$ and any measurable function $F: Q \rightarrow \mathbb{R}$,

$$
\int_{\delta^{-1}(s) \cap Q} F d \mathcal{H}_{d_{c c}}^{3}=\int_{\delta^{-1}(s) \cap Q} F \cdot\|\nabla \delta\|_{\mathbb{R}^{3}}^{-1} d \mathcal{H}_{\mathbb{R}^{3}}^{2} .
$$

The general version of the Heisenberg divergence theorem (c.f. [?, Corollary 7.7]) takes a particularly simple form when adapted to our setting.

Proposition 3.4. Let $c: U \rightarrow \mathbb{R}$ be a $\mathcal{C}^{1}$-function and let $-s_{0}<s<t<s_{0}$. Then the vector field $c N: U \rightarrow \mathbb{R}^{3}$ satisfies

$$
\int_{Q_{s, t}} \operatorname{div}_{\mathrm{H}}(c N) d \mathcal{L}^{3}=\int_{\delta^{-1}(t) \cap Q} c d \mathcal{H}_{d_{c c}}^{3}-\int_{\delta^{-1}(s) \cap Q} c d \mathcal{H}_{d_{c c}}^{3},
$$

Proof. Let us start with $V=a X_{1}+b X_{2}: U \rightarrow \mathbb{R}^{3}$ being any $\mathcal{C}^{1}$-smooth vector field with values in the horizontal distribution. Applying the Euclidean divergence theorem, Lemma 3.3 and calculating, we see that

$$
\begin{aligned}
\int_{Q_{s, t}} \operatorname{div}_{\mathrm{H}} V d \mathcal{L}^{3} & =\int_{Q_{s, t}} \operatorname{div}\left(\left(a, b, 2 a x_{2}-2 b x_{1}\right)\right) d \mathcal{L}^{3} \\
& \left.=\int_{\partial\left(Q_{s, t}\right)}\left\langle\left(a, b, 2 a x_{2}-2 b x_{1}\right), \mu\right)\right\rangle_{\mathbb{R}^{3}} d \mathcal{H}_{\mathbb{R}^{3}}^{2} \\
& =\int_{\partial\left(Q_{s, t}\right)}\left\langle V, \mu_{\mathrm{H}}\right\rangle_{\mathrm{H}} d \mathcal{H}_{\mathbb{R}^{3}}^{2}
\end{aligned}
$$

We are interested in the particular case $V=c N$. Using formula (3.5), Lemma 3.3, the fact that $\|N\|_{H}=1$ and formula (3.8), we have that

$$
\begin{aligned}
\int_{Q_{s, t}} \operatorname{div}_{\mathrm{H}}(c N) d \mathcal{L}^{3} & =\int_{\partial_{f} Q_{s, t}}\langle c N, N\rangle_{\mathrm{H}}\|\nabla \delta\|_{\mathbb{R}^{3}}^{-1} d \mathcal{H}_{\mathbb{R}^{3}}^{2}-\int_{\partial_{i} Q_{s, t}}\langle c N, N\rangle_{\mathrm{H}}\|\nabla \delta\|_{\mathbb{R}^{3}}^{-1} d \mathcal{H}_{\mathbb{R}^{3}}^{2} \\
& =\int_{\delta^{-1}(t) \cap Q} c d \mathcal{H}_{d_{c c}}^{3}-\int_{\delta^{-1}(s) \cap Q} c d \mathcal{H}_{d_{c c}}^{3} .
\end{aligned}
$$

as desired.

3.2. Derivatives and iterated divergences. In this section, we investigate the smoothness of the function

$$
\epsilon \mapsto \mathcal{L}^{3}\left(\mathcal{T}_{\mathbb{H}}\left(B_{0}, \bar{\Omega}, \epsilon\right)\right)
$$

on the interval $\left[0, s_{0}\right)$ by relating the function to the iterated divergences of $\delta$.

For each integer $i \geq 0$, we define the function $\operatorname{div}_{\mathrm{H}}{ }^{(i)} \nabla_{H} \delta: U \rightarrow \mathbb{R}$ by

$$
\begin{aligned}
& \operatorname{div}_{\mathrm{H}}{ }^{(0)} \nabla_{H} \delta=1, \\
& \operatorname{div}_{\mathrm{H}}{ }^{(i+1)} \nabla_{H} \delta=\operatorname{div}_{\mathrm{H}}\left(\left(\operatorname{div}_{\mathrm{H}}{ }^{(i)} \nabla_{H} \delta\right) \cdot N\right) .
\end{aligned}
$$


We will show that the sequence of derivatives $a^{(i)}:\left[0, s_{0}\right) \rightarrow \mathbb{R}$ inductively expressed by

$$
\begin{aligned}
& a^{(0)}(\epsilon)=\mathcal{L}^{3}\left(\mathcal{T}_{\mathbb{H}}\left(B_{0}, \bar{\Omega}, \epsilon\right)\right), \\
& a^{(i+1)}(\epsilon)= \begin{cases}\lim _{s \backslash 0} \frac{a^{(i)}(s)-a^{(i)}(0)}{s} & \epsilon=0, \\
\lim _{s \rightarrow 0} \frac{a^{(i)}(\epsilon+s)-a^{(i)}(\epsilon)}{s} & \epsilon>0,\end{cases}
\end{aligned}
$$

is indeed well-defined and can be expressed in terms of the above iterated divergences.

Theorem 3.5. For each integer $i \geq 1$ and number $\epsilon \in\left[0, s_{0}\right)$, the limit $a^{(i)}(\epsilon)$ exists and is given by

$$
a^{(i)}(\epsilon)=\int_{\delta^{-1}(\epsilon) \cap Q}\left(\operatorname{div}_{H}^{(i-1)} \nabla_{H} \delta\right) d \mathcal{H}_{d_{c c}}^{3}
$$

We will need the following result regarding the continuity of integrals with respect to level sets of $\delta$. The proof is just a consequence of the Euclidean divergence theorem.

Lemma 3.6. Let $F: Q \rightarrow \mathbb{R}$ be a $\mathcal{C}^{1}$ function. Then, for every $\epsilon \in\left(-s_{0}, s_{0}\right)$,

$$
\lim _{s \rightarrow \epsilon} \int_{\delta^{-1}(s) \cap Q} F d \mathcal{H}_{\mathbb{R}^{3}}^{2}=\int_{\delta^{-1}(\epsilon) \cap Q} F d \mathcal{H}_{\mathbb{R}^{3}}^{2}
$$

Proof of Theorem 3.5. To begin, note that for $\epsilon>0$,

$$
a^{(0)}(\epsilon)=\mathcal{L}^{3}(\bar{\Omega} \cap Q)+\mathcal{L}^{3}\left(Q_{\epsilon}\right),
$$

while $a^{(0)}(0)=\mathcal{L}^{3}(\bar{\Omega} \cap Q)$.

Using the Euclidean co-area formula, the continuity of the integral provided by Lemma 3.6, the mean value theorem, and the measure relationship given by (3.8), we see that for $\epsilon \geq 0$,

$$
\begin{aligned}
\lim _{s \searrow 0} \frac{\mathcal{L}^{3}\left(Q_{\epsilon, \epsilon+s}\right)}{s} & =\lim _{s \searrow 0} \frac{1}{s} \int_{Q_{\epsilon, \epsilon+s}} \frac{\|\nabla \delta\|_{\mathbb{R}^{3}}}{\|\nabla \delta\|_{\mathbb{R}^{3}}} d \mathcal{L}^{3} \\
& =\lim _{s \searrow 0} \frac{1}{s} \int_{\epsilon}^{\epsilon+s}\left(\int_{\delta^{-1}(\sigma) \cap Q}\|\nabla \delta\|_{\mathbb{R}^{3}}^{-1} d \mathcal{H}_{\mathbb{R}^{3}}^{2}\right) d \sigma \\
& =\int_{\delta^{-1}(\epsilon) \cap Q}\|\nabla \delta\|_{\mathbb{R}^{3}}^{-1} d \mathcal{H}_{\mathbb{R}^{3}}^{2} \\
& =\int_{\delta^{-1}(\epsilon) \cap Q} d \mathcal{H}_{d_{c c}}^{3} .
\end{aligned}
$$

Setting $\epsilon=0$ above now shows that

$$
a^{(1)}(0)=\int_{\delta^{-1}(0) \cap Q} d \mathcal{H}_{d_{c c}}^{3}=\mathcal{H}_{d_{c c}}^{3}(\partial \Omega \cap Q) .
$$

When $0<\epsilon<s_{0}$, a similar argument for $s \nearrow 0$ now implies that

$$
a^{(1)}(\epsilon)=\int_{\delta^{-1}(\epsilon) \cap Q} d \mathcal{H}_{d_{c c}}^{3} .
$$

We now assume the inductive hypothesis that for an integer $i \geq 1$ and all $0 \leq \epsilon<s_{0}$,

$$
a^{(i)}(\epsilon)=\int_{\delta^{-1}(\epsilon) \cap Q}\left(\operatorname{div}_{\mathrm{H}}{ }^{(i-1)} \nabla_{\mathrm{H}} \delta\right) d \mathcal{H}_{d_{c c}}^{3}
$$


It follows that

$$
\begin{aligned}
& \lim _{s \searrow 0} \frac{a^{(i)}(\epsilon+s)-a^{(i)}(\epsilon)}{s} \\
& \quad=\lim _{s \searrow 0} \frac{1}{s}\left(\int_{\delta^{-1}(\epsilon+s) \cap Q}\left(\operatorname{div}_{\mathrm{H}}{ }^{(i-1)} \nabla_{\mathrm{H}} \delta\right) d \mathcal{H}_{d_{c c}}^{3}-\int_{\delta^{-1}(\epsilon) \cap Q}\left(\operatorname{div}_{\mathrm{H}}{ }^{(i-1)} \nabla_{\mathrm{H}} \delta\right) d \mathcal{H}_{d_{c c}}^{3}\right) .
\end{aligned}
$$

Proposition 3.4 and the definition of the iterated divergences now yield

$$
\lim _{s \searrow 0} \frac{a^{(i)}(\epsilon+s)-a^{(i)}(\epsilon)}{s}=\lim _{s \searrow 0} \frac{1}{s} \int_{Q_{\epsilon, \epsilon+s}}\left(\operatorname{div}_{\mathrm{H}}{ }^{(i)} \nabla_{\mathrm{H}} \delta\right) d \mathcal{L}^{3} .
$$

Using the same argument that led to (3.13), we conclude that

$$
\lim _{s \searrow 0} \frac{a^{(i)}(\epsilon+s)-a^{(i)}(\epsilon)}{s}=\int_{\delta^{-1}(\epsilon) \cap Q}\left(\operatorname{div}_{\mathrm{H}}{ }^{(i)} \nabla_{\mathrm{H}} \delta\right) d \mathcal{H}_{d_{c c}}^{3} .
$$

Using a similar line of reasoning in the case that $\epsilon>0$ and $s \nearrow 0$, we now conclude that for all $\epsilon \in\left[0, s_{0}\right)$

$$
a^{(i+1)}(\epsilon)=\int_{\delta^{-1}(\epsilon) \cap Q}\left(\operatorname{div}_{\mathrm{H}}^{(i)} \nabla_{\mathrm{H}} \delta\right) d \mathcal{H}_{d_{c c}}^{3}
$$

as desired.

\section{Calculating the iterated Divergences}

4.1. A recursive formula. Now that we have related the iterated divergences to the derivatives of the volume function, it behoves us to calculate the iterated divergences. The goal of this section is to show that all iterated divergences can be expressed using second-order derivatives of the signed distance function $\delta$, although a priori $\operatorname{div}_{\mathrm{H}}{ }^{(i)} \nabla_{H} \delta$ involves $(i+1)^{\text {st }}$-order derivatives.

To simplify the notation in the coming computation, in this section we will denote the composition of vector fields $X_{i}\left(X_{j}\right)$ by $X_{i j}$ and $X_{i}\left(X_{j}\left(X_{k}\right)\right)$ by $X_{i j k}$, for $i, j, k \in\{1,2,3\}$. Products of vector fields will only be used once the vector fields have been applied to a function, namely $\delta$. For example,

$$
\left(X_{i} \delta\right)\left(X_{j k} \delta\right)=\left(X_{i} \delta\right)\left(X_{j}\left(X_{k} \delta\right)\right)
$$

We give here the proof of Theorem 1.2. The basic idea is to differentiate the eikonal equation to find relationships between various first, second, and third order derivatives of the signed distance function.

Lemma 4.1. The following relations hold:

$$
\begin{gathered}
\left\{\begin{array}{l}
\left(X_{1} \delta\right)\left(X_{11} \delta\right)+\left(X_{2} \delta\right)\left(X_{12} \delta\right)=0 \\
\left(X_{1} \delta\right)\left(X_{21} \delta\right)+\left(X_{2} \delta\right)\left(X_{22} \delta\right)=0 \\
\left(X_{1} \delta\right)\left(X_{31} \delta\right)+\left(X_{2} \delta\right)\left(X_{32} \delta\right)=0 .
\end{array}\right. \\
\left\{\begin{array}{l}
\left(X_{11} \delta\right)\left(X_{22} \delta\right)=\left(X_{21} \delta\right)\left(X_{12} \delta\right), \\
\left(X_{11} \delta\right)\left(X_{32} \delta\right)=\left(X_{31} \delta\right)\left(X_{12} \delta\right), \\
\left(X_{21} \delta\right)\left(X_{32} \delta\right)=\left(X_{31} \delta\right)\left(X_{22} \delta\right) .
\end{array}\right. \\
\left\{\begin{array}{l}
\left(X_{11} \delta\right)^{2}+\left(X_{1} \delta\right)\left(X_{111} \delta\right)+\left(X_{12} \delta\right)^{2}+\left(X_{2} \delta\right)\left(X_{112} \delta\right)=0, \\
\left(X_{21} \delta\right)^{2}+\left(X_{1} \delta\right)\left(X_{221} \delta\right)+\left(X_{22} \delta\right)^{2}+\left(X_{2} \delta\right)\left(X_{222} \delta\right)=0 \\
\left(X_{31} \delta\right)^{2}+\left(X_{1} \delta\right)\left(X_{331} \delta\right)+\left(X_{32} \delta\right)^{2}+\left(X_{2} \delta\right)\left(X_{332} \delta\right)=0 .
\end{array}\right.
\end{gathered}
$$




$$
\begin{gathered}
\left\{\begin{array}{c}
\left(X_{11} \delta\right)\left(X_{31} \delta\right)+\left(X_{1} \delta\right)\left(X_{131} \delta\right)+\left(X_{12} \delta\right)\left(X_{32} \delta\right)+\left(X_{2} \delta\right)\left(X_{132} \delta\right)=0 \\
\left(X_{21} \delta\right)\left(X_{31} \delta\right)+\left(X_{1} \delta\right)\left(X_{231} \delta\right)+\left(X_{22} \delta\right)\left(X_{32} \delta\right)+\left(X_{2} \delta\right)\left(X_{232} \delta\right)=0
\end{array}\right. \\
X_{33} \delta=\frac{1}{4}\left(X_{321} \delta-X_{312} \delta\right) . \\
\left\{\begin{array}{l}
8 X_{32} \delta=-X_{122} \delta+X_{221} \delta \\
8 X_{31} \delta=-X_{112} \delta+X_{211} \delta .
\end{array}\right.
\end{gathered}
$$

Proof. The eikonal equation (2.2) can be stated as

$$
\left(X_{1} \delta\right)^{2}+\left(X_{2} \delta\right)^{2}=1 .
$$

Therefore (4.1) follows from differentiating (4.7), by $X_{1}, X_{2}$, and $X_{3}$.

The equations in (4.1) show that the $3 \times 2$ matrix whose entries are $X_{i j}$, with $i=1,2,3$ and $j=1,2$, has a non-trivial kernel. Therefore this matrix has rank at most 1 , and the equations in (4.2) follow.

For (4.3), it is sufficient to differentiate the $i^{\text {th }}$ equation of (4.1) by $X_{i}$, for $i=1,2,3$. Similarly, the equations in (4.4) follow from differentiating the third equation of (4.1) by $X_{1}$ and $X_{2}$.

Finally, (4.5) and (4.6) hold because $X_{3}$ commutes with both $X_{1}$ and $X_{2}$, indeed:

$$
X_{33} \delta=-\frac{1}{4} X_{3}\left(X_{12} \delta-X_{21} \delta\right)=\frac{1}{4}\left(-X_{312} \delta+X_{321} \delta\right),
$$

and

$$
\begin{aligned}
2 X_{32} \delta & =X_{32} \delta+X_{23} \delta=-\frac{1}{4}\left(X_{12}-X_{21}\right) X_{2} \delta+X_{2}\left(\frac{-X_{12} \delta+X_{21} \delta}{4}\right) \\
& =\frac{1}{4}\left(-X_{122} \delta+X_{212} \delta-X_{212} \delta+X_{221} \delta\right) .
\end{aligned}
$$

The second equation of (4.6) is obtained in an analogous way.

Recall that the embedded horizontal normal $N$ is equal to the horizontal gradient of $\delta$. Hence, by definition, for $i \geq 1$,

$$
\operatorname{div}_{\mathrm{H}}{ }^{(i)} \nabla_{H} \delta=\operatorname{div}_{\mathrm{H}}\left(\left(\operatorname{div}_{\mathrm{H}}{ }^{(i-1)} \nabla_{H} \delta\right) \cdot \nabla_{\mathrm{H}} \delta\right)=\left(\operatorname{div}_{\mathrm{H}}{ }^{(i-1)} \nabla_{H} \delta\right) \Delta_{\mathrm{H}} \delta+\left\langle\nabla_{\mathrm{H}}\left(\operatorname{div}_{\mathbb{H}}^{(i-1)} \nabla_{H} \delta\right), \nabla_{\mathrm{H}} \delta\right\rangle
$$

where $\Delta_{\mathrm{H}} \delta$ is the horizontal Laplacian of $\delta$, defined by

$$
\Delta_{\mathrm{H}} \delta:=\operatorname{div}_{\mathrm{H}}\left(\nabla_{\mathrm{H}} \delta\right)
$$

The second part of the above expression behaves very nicely. Indeed, the operator $g$ defined by

$$
g(\alpha):=\left\langle\nabla_{\mathrm{H}} \alpha, \nabla_{\mathrm{H}} \delta\right\rangle,
$$

is linear and satisfies the Leibniz rule, i.e., given another differentiable function $\beta: U \rightarrow \mathbb{R}$,

$$
\begin{aligned}
& g(\alpha+\beta)=g(\alpha)+g(\beta), \\
& g(\alpha \beta)=g(\alpha) \beta+\alpha g(\beta) .
\end{aligned}
$$

With this notation in hand, we may write

$$
\operatorname{div}_{\mathrm{H}}{ }^{(i)} \nabla_{H} \delta=A\left(\operatorname{div}_{\mathrm{H}}{ }^{(i-1)} \nabla_{H} \delta\right)+g\left(\operatorname{div}_{\mathrm{H}}{ }^{(i-1)} \nabla_{H} \delta\right) .
$$


Lemma 4.2. The following relations hold:

$$
\begin{aligned}
& g(1)=0, \\
& g(A)=B+2 C-A^{2}, \\
& g(B)=0, \\
& g(C)=D-A C, \\
& g(D)=-E, \\
& g(E)=-2 A E+2 C D .
\end{aligned}
$$

Proof. The equality (4.9) follows from the Leibniz rule. For (4.10), we calculate

$$
\begin{aligned}
g(A)= & \left\langle\nabla_{\mathrm{H}}\left(X_{11} \delta+X_{22} \delta\right), \nabla_{\mathrm{H}} \delta\right\rangle=\left(X_{1} \delta\right)\left(X_{111} \delta+X_{122} \delta\right)+\left(X_{2} \delta\right)\left(X_{211} \delta+X_{222} \delta\right) \\
\stackrel{(4.3)}{=} & -\left(X_{11} \delta\right)^{2}-\left(X_{12} \delta\right)^{2}-\left(X_{2} \delta\right)\left(X_{112} \delta\right) \\
& -\left(X_{21} \delta\right)^{2}-\left(X_{1} \delta\right)\left(X_{221} \delta\right)-\left(X_{22} \delta\right)^{2}+\left(X_{1} \delta\right)\left(X_{122} \delta\right)+\left(X_{2} \delta\right)\left(X_{211} \delta\right) \\
= & -\left(X_{11}^{2} \delta+X_{22}^{2} \delta+2\left(X_{12} \delta\right)\left(X_{21} \delta\right)\right)-\left(X_{21}^{2} \delta+X_{12}^{2} \delta-2\left(X_{12} \delta\right)\left(X_{21} \delta\right)\right) \\
& -\left(X_{1} \delta\right)\left(-X_{122} \delta+X_{221} \delta\right)+\left(X_{2} \delta\right)\left(-X_{112} \delta+X_{211} \delta\right) \\
& \stackrel{(4.2),(4.6)}{=}-\left(X_{11} \delta+X_{22} \delta\right)^{2}-\left(-4 X_{3} \delta\right)^{2}-8\left(X_{1} \delta\right)\left(X_{32} \delta\right)+8\left(X_{2} \delta\right)\left(X_{31} \delta\right) \\
= & -A^{2}+B+2 C .
\end{aligned}
$$

For (4.11),

$$
g(B)=-\left\langle\nabla_{\mathrm{H}}\left(X_{3} \delta\right)^{2}, \nabla_{\mathrm{H}} \delta\right\rangle=-2\left(X_{1} \delta\right)\left(X_{3} \delta\right)\left(X_{13} \delta\right)-2\left(X_{2} \delta\right)\left(X_{3} \delta\right)\left(X_{23} \delta\right) \stackrel{(4.1)}{=} 0 .
$$

For (4.12),

$$
\begin{aligned}
g(C)= & -4\left(\left\langle\nabla_{\mathrm{H}}\left(\left(X_{1} \delta\right)\left(X_{32} \delta\right)\right), \nabla_{\mathrm{H}} \delta\right\rangle-\left\langle\nabla_{\mathrm{H}}\left(\left(X_{2} \delta\right)\left(X_{31} \delta\right)\right), \nabla_{\mathrm{H}} \delta\right\rangle\right) \\
= & -4\left(X_{32} \delta\right)\left(\left(X_{1} \delta\right)\left(X_{11} \delta\right)+\left(X_{2} \delta\right)\left(X_{21} \delta\right)\right)+4\left(X_{31} \delta\right)\left(\left(X_{1} \delta\right)\left(X_{12} \delta\right)+\left(X_{2} \delta\right)\left(X_{22} \delta\right)\right) \\
& -4\left(X_{1} \delta\right)\left(\left(X_{1} \delta\right)\left(X_{132} \delta\right)+\left(X_{2} \delta\right)\left(X_{232} \delta\right)\right)+4\left(X_{2} \delta\right)\left(\left(X_{1} \delta\right)\left(X_{131} \delta\right)+\left(X_{2} \delta\right)\left(X_{231} \delta\right)\right) \\
(4.1),(4.4)-16\left(X_{3} \delta\right)\left(\left(X_{1} \delta\right)\left(X_{31} \delta\right)+\left(X_{2} \delta\right)\left(X_{32} \delta\right)\right) & \quad-4\left(X_{1} \delta\right)\left(\left(X_{1} \delta\right)\left(X_{123} \delta-X_{213} \delta\right)-\left(X_{21} \delta\right)\left(X_{31} \delta\right)-\left(X_{22} \delta\right)\left(X_{32} \delta\right)\right) \\
& +4\left(X_{2} \delta\right)\left(\left(X_{2} \delta\right)\left(X_{213} \delta-X_{123} \delta\right)-\left(X_{11} \delta\right)\left(X_{31} \delta\right)-\left(X_{12} \delta\right)\left(X_{32} \delta\right)\right) \\
\stackrel{(4.5)}{=} & 16\left(\left(X_{1} \delta\right)^{2}+\left(X_{2} \delta\right)^{2}\right)\left(X_{33} \delta\right) \\
& +4\left(X_{1} \delta\right)\left(\left(X_{21} \delta\right)\left(X_{31} \delta\right)+\left(X_{22} \delta\right)\left(X_{32} \delta\right)\right)-4\left(X_{2} \delta\right)\left(\left(X_{11} \delta\right)\left(X_{31} \delta\right)+\left(X_{12} \delta\right)\left(X_{32} \delta\right)\right) \\
& +4\left(X_{1} \delta\right)\left(X_{11} \delta\right)\left(X_{32} \delta\right)-4\left(X_{1} \delta\right)\left(X_{11} \delta\right)\left(X_{32} \delta\right)+4\left(X_{2} \delta\right)\left(X_{22} \delta\right)\left(X_{31} \delta\right)-4\left(X_{2} \delta\right)\left(X_{22} \delta\right)\left(X_{31} \delta\right) \\
= & D+4\left[\left(X_{1} \delta\right)\left(X_{32} \delta\right)-\left(X_{2} \delta\right)\left(X_{31} \delta\right)\right]\left(X_{11} \delta+X_{22} \delta\right) \\
& -4\left(X_{32} \delta\right)\left[\left(X_{1} \delta\right)\left(X_{11} \delta\right)+\left(X_{2} \delta\right)\left(X_{12} \delta\right)\right]+4\left(X_{31} \delta\right)\left[\left(X_{1} \delta\right)\left(X_{21} \delta\right)+\left(X_{2} \delta\right)\left(X_{22} \delta\right)\right] \\
\stackrel{(4.1)}{=} & D-A C .
\end{aligned}
$$

For (4.13),

$$
g(D)=16\left\langle\nabla_{\mathrm{H}}\left(X_{33} \delta\right), \nabla_{\mathrm{H}} \delta\right\rangle=16\left[\left(X_{1} \delta\right)\left(X_{133} \delta\right)+\left(X_{2} \delta\right)\left(X_{233} \delta\right)\right] \stackrel{(4.3)}{=}-E .
$$


For (4.14),

$$
\begin{aligned}
g(E)= & 16\left\langle\nabla_{\mathrm{H}}\left(\left(X_{31} \delta\right)^{2}+\left(X_{32} \delta\right)^{2}\right), \nabla_{\mathrm{H}} \delta\right\rangle \\
= & 32\left(X_{1} \delta\right)\left[\left(X_{31} \delta\right)\left(X_{131} \delta\right)+\left(X_{32} \delta\right)\left(X_{132} \delta\right)\right]+32\left(X_{2} \delta\right)\left[\left(X_{31} \delta\right)\left(X_{231} \delta\right)+\left(X_{32} \delta\right)\left(X_{232} \delta\right)\right] \\
\stackrel{(4.4)}{=} & 32\left(X_{31} \delta\right)\left[-\left(X_{11} \delta\right)\left(X_{31} \delta\right)-\left(X_{12} \delta\right)\left(X_{32} \delta\right)-\left(X_{2} \delta\right)\left(X_{123} \delta-X_{213} \delta\right)\right] \\
& \quad+32\left(X_{32} \delta\right)\left[-\left(X_{21} \delta\right)\left(X_{31} \delta\right)-\left(X_{22} \delta\right)\left(X_{32} \delta\right)+\left(X_{1} \delta\right)\left(X_{123} \delta-X_{213} \delta\right)\right] \\
& \stackrel{(4.5)}{=}-128\left(X_{33} \delta\right)\left[\left(X_{1} \delta\right)\left(X_{32} \delta\right)-\left(X_{2} \delta\right)\left(X_{31} \delta\right)\right]-32\left(X_{11} \delta\right)\left(X_{31} \delta\right)^{2}-32\left(X_{22} \delta\right)\left(X_{32} \delta\right)^{2} \\
& -32\left(X_{31} \delta\right)\left(X_{32} \delta\right)\left[X_{12} \delta+X_{21} \delta\right]+32\left(X_{11} \delta\right)\left(X_{32} \delta\right)^{2}-32\left(X_{11} \delta\right)\left(X_{32} \delta\right)^{2} \\
& +32\left(X_{22} \delta\right)\left(X_{31} \delta\right)^{2}-32\left(X_{22} \delta\right)\left(X_{31} \delta\right)^{2} \\
= & 2 C D-32\left(X_{11} \delta+X_{22} \delta\right)\left[\left(X_{31} \delta\right)^{2}+\left(X_{32} \delta\right)^{2}\right]+32\left(X_{31} \delta\right)\left[\left(X_{22} \delta\right)\left(X_{31} \delta\right)-\left(X_{21} \delta\right)\left(X_{32} \delta\right)\right] \\
& +32\left(X_{32} \delta\right)\left[\left(X_{11} \delta\right)\left(X_{32} \delta\right)-\left(X_{12} \delta\right)\left(X_{31} \delta\right)\right] \\
& \stackrel{(4.2)}{=} 2 C D-2 A E .
\end{aligned}
$$

Proof of Theorem 1.2. The first four iterated divergences are easy to calculate using (4.8).

We proceed by induction on $j$, having already proven the desired result when $j=2$. Assume that (1.2) and(1.3) hold for some $j \geq 2$. Now,

$$
\begin{aligned}
\operatorname{div}_{\mathrm{H}}^{(2(j+1)-1)} \nabla_{H} \delta= & \operatorname{div}_{\mathrm{H}}^{(2 j+1)} \nabla_{H} \delta=A \operatorname{div}_{\mathrm{H}}{ }^{(2 j)} \nabla_{H} \delta+g\left(\operatorname{div}_{\mathrm{H}}{ }^{(2 j)} \nabla_{H} \delta\right) \\
\stackrel{(1.3)}{=} & A B^{j-2}\left(\operatorname{div}_{\mathrm{H}}{ }^{(4)} \nabla_{H} \delta+2(j-2)(A D-E)\right) \\
& +B^{j-2} g\left(\operatorname{div}_{\mathrm{H}}^{(4)} \nabla_{H} \delta+2(j-2)(A D-E)\right) \\
= & A B^{j-2}\left(B^{2}+2 B C+2 A D-2 E+2(j-2)(A D-E)\right) \\
& +B^{j-2} g\left(B^{2}+2 B C+2 A D-2 E+2(j-2)(A D-E)\right) \\
= & A B^{j}+2 A B^{j-1} C+2(j-1) A^{2} B^{j-2} D-2(j-1) A B^{j-2} E \\
& +B^{j-2} g\left(B^{2}+2 B C+2(j-1)(A D-E)\right) \\
= & A B^{j}+2 A B^{j-1} C+2(j-1) A^{2} B^{j-2} D-2(j-1) A B^{j-2} E \\
& +B^{j-2}(2 B D-2 A B C+2(j-1) A g(D)+2(j-1) D g(A)-2(j-1) g(E)) \\
= & A B^{j}+2 A B^{j-1} C+2(j-1) A^{2} B^{j-2} D-2(j-1) A B^{j-2} E+2 B^{j-1} D \\
& -2 A B^{j-1} C-2(j-1) A B^{j-2} E-2(j-1) A^{2} B^{j-2} D+4(j-1) B^{j-2} C D \\
& +2(j-1) B^{j-1} D+4(j-1) A B^{j-2} E-4(j-1) B^{j-2} C D \\
= & B^{j-1}(A B+2 D+2(j-1) D) \\
= & B^{(j+1)-2}\left(\operatorname{div}_{\mathrm{H}}^{(3)} \nabla_{H} \delta+2((j+1)-2) D\right) . \\
& -2 .
\end{aligned}
$$

Finally, using the result just obtained for $\operatorname{div}_{\mathrm{H}}{ }^{(2 j+1)} \nabla_{H} \delta$, we get the following 


$$
\begin{aligned}
\operatorname{div}_{\mathrm{H}}{ }^{(2(j+1))} \nabla_{H} \delta & =\operatorname{div}_{\mathrm{H}}{ }^{(2 j+2)} \nabla_{H} \delta=A \operatorname{div}_{\mathrm{H}}{ }^{(2 j+1)} \nabla_{H} \delta+g\left(\operatorname{div}_{\mathrm{H}}{ }^{(2 j+1)} \nabla_{H} \delta\right) \\
& =A B^{j-1}\left(\operatorname{div}_{\mathrm{H}}^{(3)} \nabla_{H} \delta+2(j-1) D\right)+B^{j-1} g\left(\operatorname{div}_{\mathrm{H}}{ }^{(3)} \nabla_{H} \delta+2(j-1) D\right) \\
& =A B^{j-1}(A B+2 D+2(j-1) D)+B^{j-1} g(A B+2 D+2(j-1) D) \\
& =A B^{j-1}(A B+2 j D)+B^{j-1} g(A B+2 j D) \\
& =A^{2} B^{j}+2 j A B^{j-1} D+B^{j} g(A)+2 j B^{j-1} g(D) \\
& =A^{2} B^{j}+2 j A B^{j-1} D-A^{2} B^{j}+2 B^{j} C+B^{j+1}-2 B^{j-1} E \\
& =B^{j-1}\left(B^{2}+2 B C+2 j A D-2 j E\right) \\
& =B^{j-1}\left(B^{2}+2 B C+2 A D-2 E+2(j-1)(A D-E)\right) \\
& =B^{(j+1)-2}\left(\operatorname{div}_{\mathrm{H}}{ }^{(4)} \nabla_{H} \delta+2((j+1)-2)(A D-E)\right) .
\end{aligned}
$$

This completes the induction argument.

4.2. Analyticity of the volume function. As an application of the recursive formula for the iterated divergences found in the previous section, we show that the volume function is analytic. This completes the proof of the main theorem of this paper.

Theorem 4.3. For $\epsilon \geq 0$, let $\mathcal{T}_{\mathbb{H}}\left(B_{0}, \bar{\Omega}, \epsilon\right)$ be a localized Heisenberg $\epsilon$-neighborhood of $\Omega$ as defined in Section 3.1. Then the function $a^{(0)}:\left[0, s_{0}\right] \rightarrow \mathbb{R}$ defined by

$$
a^{(0)}(\epsilon)=\mathcal{L}^{3}\left(\mathcal{T}_{\mathbb{H}}\left(B_{0}, \bar{\Omega}, \epsilon\right)\right)
$$

is real-analytic, and has a power series expansion at $\epsilon=0$ given by

$$
a^{(0)}(\epsilon)=\mathcal{L}^{3}(\bar{\Omega} \cap Q)+\sum_{i=1}^{\infty}\left(\int_{\partial \Omega \cap Q}\left(\operatorname{div}_{\mathrm{H}}{ }^{(i-1)} \nabla_{H} \delta\right) d \mathcal{H}_{d_{c c}}^{3}\right) \frac{\epsilon^{i}}{i !} .
$$

Proof. We estimate $a^{(i)}(\epsilon)$ for a positive even integer $i=2 j$ and $\epsilon \in\left[0, s_{0}\right]$; a similar argument is valid when $i$ is odd. By Theorem 3.5,

$$
\begin{aligned}
\left|a^{(i)}(\epsilon)\right| & \leq \int_{\delta^{-1}(\epsilon) \cap Q}\left|\operatorname{div}_{\mathrm{H}}{ }^{(2 j-1)} \nabla_{H} \delta\right| d \mathcal{H}_{d_{c c}}^{3} \\
& =\int_{\delta^{-1}(\epsilon) \cap Q}\left|A B^{j-1}+2(j-1) B^{j-2} D\right| d \mathcal{H}_{d_{c c}}^{3} \\
& \leq \int_{\delta^{-1}(\epsilon) \cap Q}(|A|+|B|)^{j}+2(j-1)(1+|B|+|D|)^{j} d \mathcal{H}_{d_{c c}}^{3} .
\end{aligned}
$$

Due to the smoothness of $\delta$, there exists a constant $L>0$ such that the last integrand is bounded by $L^{j}$ on compact sets. Since $\delta^{-1}(\epsilon) \cap Q$ is included in a compact set, we have that there exists a constant $M>0$ satisfying

$$
\sup _{\epsilon \in\left[0, s_{0}\right]}\left|a^{(i)}(\epsilon)\right| \leq M^{j}
$$

This and its counter-part for odd $i$ quickly imply the analyticity of $a^{(0)}$. 


\section{AN EXAMPLE AND FINAL REMARKS}

In this section we present an example in which we can calculate the volume of a localized Heisenberg $\epsilon$-tube. The basic idea is to view the localizing set $Q$ as foliated by integral curves of the flow of the horizontal normal. It is in general non-trivial to calculate the signed distance function to a given set, the embedded horizontal normal, or its flow. However, general results from [?] and [?] give a formula for the integral curves of the embedded horizontal normal. The embedded horizontal normal itself can then be obtained by differentiation, and in certain cases (such as the coming example), a change of coordinates can be used to determine the signed distance function.

Example 5.1. We now consider the half-space

$$
\mathbb{H}_{x_{3}^{-}}=\left\{\left(x_{1}, x_{2}, x_{3}\right) \in \mathbb{H}: x_{3}<0\right\}
$$

with boundary

$$
\partial \mathbb{H}_{x_{3}^{-}}=\left\{\left(x_{1}, x_{2}, x_{3}\right) \in \mathbb{H}: x_{3}=0\right\}
$$

which has a single characteristic point at $(0,0,0)$.

For $0<r<R$, we consider the annulus

$$
B_{0}=\left\{\left(x_{1}, x_{2}, 0\right) \in \mathbb{R}^{3}: r^{2}<x_{1}^{2}+x_{2}^{2}<R^{2}\right\},
$$

and denote by $U \subseteq \mathbb{H}$ an open set that contains the annulus $B_{0}$ and on which the signed-distance to $\partial \mathbb{H}_{x_{3}^{-}}$function $\delta$ is $\mathcal{C}^{\infty}$-smooth. Denote by $N: U \rightarrow \mathbb{R}^{3}$ the embedded horizontal normal. By [?, Proposition 3.1], for $g=\left(g_{1}, g_{2}, 0\right) \in \partial \mathbb{H}_{x_{3}^{-}} \cap U$, the solution $\varphi_{g}=\left(\varphi_{1}, \varphi_{2}, \varphi_{3}\right)$ of the Cauchy problem

$$
\left\{\begin{array}{l}
\dot{\varphi}(s)=N(\varphi(s)), \\
\varphi(0)=g
\end{array}\right.
$$

is given by

$$
\varphi_{g}(s)=\left(\begin{array}{l}
\varphi_{1}(s) \\
\varphi_{2}(s) \\
\varphi_{3}(s)
\end{array}\right)=\left(\begin{array}{l}
\frac{g_{1}}{2}\left(1+\cos \left(\frac{2 s}{|g|}\right)\right)+\frac{g_{2}}{2} \sin \left(\frac{2 s}{|g|}\right) \\
\frac{g_{2}}{2}\left(1+\cos \left(\frac{2 s}{|g|}\right)\right)-\frac{g_{1}}{2} \sin \left(\frac{2 s}{|g|}\right) \\
\frac{|g|^{2}}{2}\left(\frac{2 s}{|g|}+\sin \left(\frac{2 s}{|g|}\right)\right) .
\end{array}\right),
$$

where $|g|=\left(g_{1}^{2}+g_{2}^{2}\right)^{1 / 2}$. Moreover, there is a number $s_{0}>0$ such that for any $g \in U_{0}$, the solution above exists on the interval $\left[-s_{0}, s_{0}\right]$.

We consider the localizing set $Q$ generated by the set $B_{0}$ and depth $s_{0}$, i.e., we localize using the annulus and the flow defined above. We wish to calculate, for $0<\epsilon<s_{0}$, the volume of the resulting tube $\mathcal{T}_{\mathbb{H}}\left(B_{0}, \overline{\mathbb{H}}_{x_{3}^{-}}, \epsilon\right)$.

We introduce a new coordinate system of $Q$, as in [?]. For each point $\left(x_{1}, x_{2}, x_{3}\right) \in Q$, we may find a $g \in B_{0}$ and $s \in\left[-s_{0}, s_{0}\right]$ so that

$$
\left(x_{1}, x_{2}, x_{3}\right)=\varphi_{g}(s) .
$$

We first express $g$ in polar coordinates as

$$
g=\left(g_{1}, g_{2}, 0\right)=(\rho \cos \theta, \rho \sin \theta, 0),
$$

and then set

$$
\beta=\frac{s}{\rho} \text { and } \alpha=\theta-\beta .
$$


Our new coordinate system on $Q$ is $(\rho, \alpha, \beta)$. Writing (5.1) in these coordinates and simplifying shows that

$$
\left(x_{1}, x_{2}, x_{3}\right)=\varphi_{g}(s)=\left(\rho \cos (\beta) \cos (\alpha), \rho \cos (\beta) \sin (\alpha), \rho^{2}\left(\beta+\frac{\sin 2 \beta}{2}\right)\right) .
$$

By Proposition 3.1,

$$
\delta\left(x_{1}, x_{2}, x_{3}\right)=s=\rho \beta .
$$

Hence, using the chain rule, we may calculate that

$$
X_{3} \delta=\frac{1}{2 \rho}, \quad\left(X_{1} \delta\right)\left(X_{32} \delta\right)-\left(X_{2} \delta\right)\left(X_{31} \delta\right)=-\frac{\cos \beta}{2 \rho^{2}(\cos \beta+\beta \sin \beta)},
$$

and

$$
X_{33} \delta=-\frac{\sin \beta}{4 \rho^{3}(\cos \beta+\beta \sin \beta)}, \quad\left(X_{31} \delta\right)^{2}+\left(X_{32} \delta\right)^{2}=\frac{\cos ^{2}(\beta)}{4 \rho^{4}(\cos \beta+\beta \sin \beta)^{2}} .
$$

Setting $\beta=0$ we can compute the functions $A, B, C, D$ and $E$ restricted to the plane $\partial \mathbb{H}_{x_{3}^{-}}$:

$$
A=D=0, \quad B=-\frac{4}{\rho^{2}}, \quad C=\frac{2}{\rho^{2}}, \quad E=\frac{4}{\rho^{4}} .
$$

Note that the Euclidean outward pointing unit normal to $\mathbb{H}_{x_{3}^{-}}$is $\nu=(0,0,1)$. Hence

$$
\|\nabla \delta\|_{\mathbb{R}^{3}}^{-1}=\sqrt{\left\langle X_{1}, \nu\right\rangle^{2}+\left\langle X_{2}, \nu\right\rangle^{2}}=2 \sqrt{x_{1}^{2}+x_{2}^{2}}=2 \rho .
$$

It follows that

$$
\int_{\partial \Omega \cap Q}\left(\operatorname{div}_{\mathrm{H}}{ }^{(0)} \nabla_{H} \delta\right) d \mathcal{H}_{d_{c c}}^{3}=\int_{\partial \Omega \cap Q}\|\nabla \delta\|_{\mathbb{R}^{3}}^{-1} d \mathcal{H}_{\mathbb{R}^{3}}^{2}=\frac{4 \pi}{3}\left(R^{3}-r^{3}\right) .
$$

Moreover, for each integer $n \geq 0$, using (1.3) with $j=n+1$ yields

$$
\begin{gathered}
\int_{\partial \Omega \cap Q}\left(\operatorname{div}_{\mathrm{H}}{ }^{(2 n+2)} \nabla_{H} \delta\right) d \mathcal{H}_{d_{c c}}^{3}=2 \int_{0}^{2 \pi} \int_{r}^{R} \rho^{2}\left(\left(\frac{-4}{\rho^{2}}\right)^{n-1}\left(\frac{-8 n}{\rho^{4}}\right)\right) d \rho d \theta \\
=\frac{4 \pi(-1)^{n} n 2^{2 n+1}}{(1-2 n)}\left(R^{1-2 n}-r^{1-2 n}\right) .
\end{gathered}
$$

Plugging these results into the statement of Theorem 4.3 gives a Taylor series expansion for the tube $\mathcal{L}^{3}\left(\mathcal{T}_{\mathbb{H}}\left(B_{0}, \overline{\mathbb{H}}_{x_{3}^{-}}, \epsilon\right)\right)$ of depth $s_{0}$, at $\epsilon=0$ : namely

$$
\begin{aligned}
\mathcal{L}^{3}\left(\mathcal{T}_{\mathbb{H}}\left(B_{0}, \overline{\mathbb{H}}_{x_{3}^{-}}, \epsilon\right)\right)= \\
\quad=\frac{4 \pi}{3}\left(R^{3}-r^{3}\right)\left(\epsilon+s_{0}\right)+4 \pi \sum_{n=0}^{\infty} \frac{(-1)^{n} 2^{2 n+1} n\left(R^{1-2 n}-r^{1-2 n}\right)}{(1-2 n)(2 n+3) !}\left(\epsilon^{2 n+3}+s_{0}^{2 n+3}\right) .
\end{aligned}
$$

It is clear that $\mathcal{L}^{3}\left(\mathcal{T}_{\mathbb{H}}\left(B_{0}, \overline{\mathbb{H}}_{x_{3}^{-}}, \epsilon\right)\right)$ blows up as $r \rightarrow 0$, and formula (5.2) provides explicitly the rate of divergence in terms of powers of $r$.

Before finishing the paper a few remarks are in order: Our first remark is about the regularity assumptions. In order to avoid technical complications we assumed a maximal degree of regularity, namely $\mathcal{C}^{\infty}$ of our surfaces and the associated defining functions. Therefore all iterated divergences are a priori well defined. However, our proofs show that the regularity $\mathcal{C}^{4}$ of the surface suffices. Indeed, this implies a $\mathcal{C}^{3}$ regularity of the normalized defining function. Let us observe that all iterated divergences are expressed as second order derivatives of the normalized distance function, 
an in our proofs we need at most first order derivatives of these expressions. This shows that for our considerations $\mathcal{C}^{4}$ smoothness is sufficient.

Let us mention,that in the Euclidean versions of Steiner's formula a much less regularity is sufficient. Indeed, the notion of positive reach [?] is enough to use in this context. It is in an interesting problem to study sets of positive reach in the context of sub-Riemannian geometries in connection with Steiner type formulae.

It is an interesting question to investigate the geometric meaning of the iterated divergences appearing in this paper. In the forthcoming article: [?] the third coefficient of the expansion is interpreted as horizontal Gauss curvature. In this paper it is shown that for regular compact surfaces with no characteristic points, the Steiner formula is reduced to a second degree polynomial. This could indicate the fact that higher order coefficients have no intrinsic geometric interpretation and are related to the presence of boundary terms or of the characteristic points.

A natural generalization of Steiner's formula in Riemannian manifolds is the tube formula of Weyl [?] which gives the volume of a tube of a sub manifold in the Riemannian context. It would be very interesting to investigate the validity of tube formulas in higher dimensional Heisenberg groups.

Mathematisches Institut, Universität Bern, Sidlerstrasse 5, CH-3012 Bern, Switzerland

E-mail address: zoltan.balogh@math.unibe.ch

E-mail address: eugenio.vecchi@math.unibe.ch

Dipartimento di Matematica, Universita di Bologna, Piazza di Porta San Donato 5, 40126 Bologna ITALY

E-mail address: fausto.ferrari@unibo.it

E-mail address: bruno.franchi@unibo.it

Department of Mathematical Sciences, Montana State University, P.O. Box 172400, Bozeman, MT 59717-2400, USA

E-mail address: kevin.wildrick@montana.edu 